\title{
A new low-cost point-of-care test for liver function has been developed
}

In resource-limited countries, patients at high risk of drug-induced liver injury are often not monitored for hepatotoxicity because of the expense and logistics of using current tests. A research team from the USA has now developed a low-cost paper-based assay that can be used to monitor levels of aspartate aminotransferase (AST) and alanine aminotransferase (ALT).

The researchers tested 223 clinical venipuncture specimens from patients with a variety of underlying medical conditions and 10 fingerstick specimens from healthy volunteers with the new assay and a standard automated method. Within $15 \mathrm{~min}$, the new assay provided a visual measurement of AST and ALT levels. The results could then be placed in one of three categories: $<3$ times the upper limit of normal (ULN), 3-5 times the ULN and $>5$ times the ULN. Results from the new test were comparable to those obtained from the automated method, even in samples up to $5 \mathrm{~h}$ old.

"These data have firmly established proof-of-concept and clinical relevance and suggest that the point-of-care fingerstick device will have a high impact on patient care in low-resource settings," say authors Nira Pollock and Jason Rolland. The authors are now planning a preliminary field study in Vietnam to evaluate the new device in 600 patients with HIV. "This platform could easily be modified to develop novel diagnostic tests for other important clinical applications," conclude Pollock and Rolland.

\section{Claire Greenhill}

Original article Pollock, N. R. et al. A paper-based multiplexed transaminase test for low-cost, point-of-care liver function testing. Sci. Transl. Med. 4, 152ra129 (2012) 